\title{
Differential gene expression pattern in early gastric cancer by an integrative systematic approach
}

\author{
SEUNGYOON NAM ${ }^{1 *}$, JIEUN LEE $^{1 *}$, SUNG-HO GOH $^{2}$, SEUNG-HYUN HONG $^{1}$, NAALEUM SONG ${ }^{1}$, \\ SANG-GEUN JANG ${ }^{3}$, IL JU CHOI ${ }^{4}$ and YEON-SU LEE ${ }^{1}$ \\ ${ }^{1}$ Cancer Genomics Branch, ${ }^{2}$ New Experimental Therapeutics Branch, ${ }^{3}$ Colorectal Cancer Branch \\ and ${ }^{4}$ Gastric Cancer Branch, Research Institute, National Cancer Center, \\ Goyang, Gyeonggi-do 410-769, Republic of Korea
}

Received April 30, 2012; Accepted July 2, 2012

DOI: $10.3892 /$ ijo.2012.1621

\begin{abstract}
To elucidate the molecular basis of early gastric cancer (EGC), the genome-wide expression pattern of cancer and normal tissues from 27 patients were analyzed by a microarray-based method. Using an integrative systematic bioinformatics approach, we classified the differentially expressed genes in EGC. Interestingly, the more highly expressed genes in EGC exhibited the most significant correlation with cell migration and metastasis. This implies that, even at the early stage of gastric cancer, the molecular properties usually observed in late-stage cancer are already present. Furthermore, we have found a novel association between the expression pattern and molecular pathways of EGC and estrogen receptor $\alpha(\mathrm{ER} \alpha)$-negative breast cancer through cross-experimental analysis. These results provide new insights into the biological properties of EGC, as well as yielding useful basic data for the study of molecular mechanisms of EGC carcinogenesis.
\end{abstract}

\section{Introduction}

Gastric cancer (GC) is the fourth most common cancer and the second leading cause of cancer-related deaths worldwide. Its prevalence is particularly high in East Asia, including countries such as China, Japan and Korea (1). The prognosis

Correspondence to: Dr Yeon-Su Lee, Cancer Genomics Branch, Research Institute, National Cancer Center, 323 Ilsan-ro, Goyang, Gyeonggi-do 410-769, Republic of Korea

E-mail: yslee2@ncc.re.kr

Dr Il Ju Choi, Gastric Cancer Branch, Research Institute, National Cancer Center, 323 Ilsan-ro, Goyang, Gyeonggi-do 410-769, Republic of Korea

E-mail: cij1224@ncc.re.kr

*Contributed equally

Key words: estrogen receptor $\alpha$, gastric cancer, metastasis, microarray, metalloproteinases of GC depends on the stage of diagnosis, as an early gastric cancer (EGC) or advanced gastric cancer (AGC) (2). Despite the surgical advances that have improved long-term survival of GC patients $(3,4)$, molecular understanding of, as well as novel molecular biomarkers for, the condition is still urgently required for EGC, as EGC may progress towards AGC (2).

To address this, several microarray analyses in GC have been performed and have identified gene expression patterns that may be useful in the prognosis and diagnosis of the cancer $(5,6)$; however, these approaches did not consider the different stages or subtypes of GC. Recent studies that did consider stage differences $(2,7,8)$ did not reveal the multiple phenotypes underlining EGC, because their primary aim was to study a handful of gene sets, which differentiate the stage differences. Accordingly, we further explored the various hidden phenotypes, functions and pathways in EGC by using an integrative systematic bioinformatics approach.

Here, we focus on molecular understanding of EGC-specific expression patterns gained by employing a systematic approach, including function and pathway, as well as crossexperiment analyses of 27 pairs of EGC tissues and their normal counterparts. Interestingly, the function and pathway analyses show that the upregulated genes in EGC tissues correlate with cell migration and metastasis, events typical of late-stage cancer. In addition, we propose a novel association between EGC and estrogen receptor $\alpha(\mathrm{ER} \alpha)$-negative breast cancer that was indicated by cross-experiment analysis, and which enables us to identify various associated phenotypes.

\section{Materials and methods}

Patients and samples. Tissue samples were prospectively collected from patients who underwent gastric surgery or gastroscopy at the National Cancer Center (NCC) Hospital between 2008 and 2009. All tissues were obtained according to the protocols approved by the Institutional Review Board, NCC for the human subject guideline of NCC (NCCNCS-08-127) that is in accordance with the principles of the Declaration of Helsinki. The samples were obtained by endoscopic biopsies from gastric cancer patients who gave informed consent to the protocol. The samples were stored at $-80^{\circ} \mathrm{C}$. The clinical and pathological features of the patients are listed in Table I. 
Table I. Clinical features for 27 patients with gastric cancer.

\begin{tabular}{|c|c|}
\hline Characteristics & No. of patients ${ }^{\mathrm{a}}$ \\
\hline Total & 27 \\
\hline Male & 20 \\
\hline Female & 7 \\
\hline \multicolumn{2}{|c|}{ Age at diagnosis (years) } \\
\hline Range & $41-78$ \\
\hline Mean $\pm \mathrm{SD}$ & $60.3 \pm 11.2$ \\
\hline \multicolumn{2}{|l|}{ 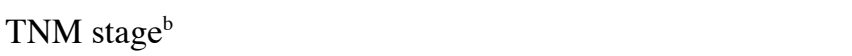 } \\
\hline \multicolumn{2}{|c|}{$\mathrm{T}$ classification } \\
\hline $\mathrm{T} 1$ & 17 \\
\hline $\mathrm{T} 2$ & 10 \\
\hline $\mathrm{T} 3$ & 0 \\
\hline \multicolumn{2}{|c|}{$\mathrm{N}$ classification } \\
\hline N0 & 13 \\
\hline N1 & 10 \\
\hline $\mathrm{N} 2$ & 2 \\
\hline N3 & 2 \\
\hline \multicolumn{2}{|c|}{ M classification } \\
\hline M0 & 27 \\
\hline M1 & 0 \\
\hline \multicolumn{2}{|c|}{ Lauren classification } \\
\hline Intestinal & 12 \\
\hline Diffuse & 7 \\
\hline Mixed & 6 \\
\hline $\mathrm{NA}^{\mathrm{c}}$ & 2 \\
\hline
\end{tabular}

${ }^{a}$ The 27 patient samples were used in microarray analysis. ${ }^{b}$ UICC/AJCC 6th edition. ${ }^{\mathrm{c} N o t}$ available of Lauren classification.

RNA extraction. Total RNA was extracted from gastric cancer and adjacent normal tissues from EGC patients using TRIzol reagent (Invitrogen, Carlsbad, CA, USA), followed by purification of the RNA using Qiagen RNeasy mini kit columns (Valencia, CA, USA) according to the manufacturer's instructions. RNA quality was evaluated using the Agilent 2100 Bioanalyzer (Agilent Technologies, Palo Alto, CA, USA) and concentration measured by Nanodrop 1000 (Thermo Scientific, Wilmington, DE, USA). Only RNAs showing distinct $18 \mathrm{~S} / 28 \mathrm{~S}$ ribosomal peak ratios of $1.5-2.0$ in the Bioanalyzer (Agilent Technologies) and 260/280 ratios of 1.8-2.1 in the Nanodrop (Thermo Scientific) analyses were accepted for further analysis.

Microarray analysis and data processing. Genome-wide gene expression was analyzed in the 27-paired EGC tissue samples using Affymetrix GeneChip Human Exon1.0 ST Array (Santa Clara, CA, USA). Target preparation and microarray processing procedures were carried out as described in the manufacturer's instructions, and raw data were deposited in the NCBI Gene Expression Omnibus (GSE30727). The data were preprocessed by a default robust multi-array average (RMA) method implemented in the Bioconductor (www.bioconductor.org)
Table II. The primer sequences used in RT-PCR.

\begin{tabular}{ll}
\hline Primer ID & \multicolumn{1}{c}{ Sequence $\left(5^{\prime} \rightarrow 3^{\prime}\right)$} \\
\hline MMP1-F & CTGGAATTGGCCACAAAGTT \\
MMP1-R & CCTTCTTTGGACTCACACCA \\
MMP3-F & CCCTGGGTCTCTTTCACTCA \\
MMP3-R & TCAAAGGACAAAGCAGGATC \\
MMP7-F & CGGATGGTAGCAGTCTAGGG \\
MMP7-R & TGAATGGATGTTCTGCCTGA \\
MMP9-F & GGGAAGATGCTGCTGTTCA \\
MMP9-R & TCAACTCACTCCGGGAACTC \\
MMP10-F & GGCTCTTTCACTCAGCCAAC \\
MMP10-R & TCCCGAAGGAACAGATTTTG \\
MMP12-F & CCTTCAGCCAGAAGAACCTG \\
MMP12-R & ACACATTTCGCCTCTCTGCT \\
MMP13-F & TTGAGCTGGACTCATTGTCG \\
MMP13-R & GGAGCCTCTCAGTCATGGAG \\
GAPDH-F & TGCACCACCAACTGCTTA \\
GAPDH-R & GGATGCAGGGATGATGTTC \\
\hline
\end{tabular}

'oligo' package. The differentially expressed genes between EGC tissues and adjacent non-cancerous gastric tissues (i.e., the up- and downregulated genes in EGC) were filtered by a fold-change cut-off of 1.5 and a P-value cut-off of 0.05 .

Functional/pathway enrichment analysis and cross-experimental analysis. We downloaded a Gene Ontology (GO) annotation file (gene_association.goa_human) and an ontology file (gene_ontology_ext.obo) from www.geneontology.org, as recommended by the BiNGO tutorial (9). In the BiNGO analysis, all options, except for filtering the IEA code, were set at default values. The false discovery rate (FDR) cut-off was 0.05 . DAVID v6.7 software (http://david.abcc.ncifcrf.gov/) was used to summarize the over-representation of the KEGG pathways (10). The gene expression signatures of up- or downregulated genes in EGC were analyzed using the L2L microarray analysis tool (http://depts.washington.edu/l21/) (11).

Reverse transcription PCR. Two micrograms of total RNA were reverse transcribed with Superscript III reverse transcriptase (Invitrogen). Reverse transcription PCR (RT-PCR) was performed using $5 \mathrm{ng} \mathrm{cDNA}$ for 1 cycle at $94^{\circ} \mathrm{C}$ for $2 \mathrm{~min}$, followed by $32-35$ cycles of $94^{\circ} \mathrm{C}$ for $20 \mathrm{sec}, 60^{\circ} \mathrm{C}$ for $40 \mathrm{sec}$ and $72^{\circ} \mathrm{C}$ for $30 \mathrm{sec}$, using gene-specific primers (Table II). Gene expression levels were analyzed by gel electrophoresis.

Hierarchical clustering. Independent additional cancer datasets were obtained from NCBI GEO (www.ncbi.nlm.nih.gov/ geo) and EBI ArrayExpress (www.ebi.ac.uk/arrayexpress): GSE19536 for ER $\alpha$-negative breast cancers (12), and the E-MTAB-62 dataset for Ewing's sarcoma, bladder cancer, small cell lung cancer and LNCaP prostate cancer cell lines (13). The up- and downregulated genes in the EGC tissues were compared with these 5 cancer types. We transformed the expression of all our EGC tissue samples, GSE19536 
Table III. The GO biological process terms associated with genes upregulated in gastric cancer tissues, relating to wound healing, cell migration and cell motility.

\begin{tabular}{lcccccl}
\hline GO-ID & P-value & $\begin{array}{c}\text { Corrected } \\
\text { P-value }\end{array}$ & $\mathrm{x}^{\mathrm{b}}$ & $\mathrm{n}^{\mathrm{c}}$ & $\mathrm{x} / \mathrm{n}(\%)$ & \\
\hline GO:0014910 & $1.54 \mathrm{E}-03$ & $1.86 \mathrm{E}-02$ & 4 & 14 & 29 & Regulation of smooth muscle cell migration \\
GO:0061041 & $5.41 \mathrm{E}-07$ & $3.15 \mathrm{E}-05$ & 11 & 44 & 25 & Regulation of wound healing \\
GO:0010595 & $4.41 \mathrm{E}-03$ & $4.25 \mathrm{E}-02$ & 5 & 29 & 17 & Positive regulation of endothelial cell migration \\
GO:0030335 & $3.69 \mathrm{E}-07$ & $2.38 \mathrm{E}-05$ & 18 & 116 & 16 & Positive regulation of cell migration \\
GO:2000147 & $3.69 \mathrm{E}-07$ & $2.38 \mathrm{E}-05$ & 18 & 116 & 16 & Positive regulation of cell motility \\
GO:0030334 & $9.12 \mathrm{E}-07$ & $4.44 \mathrm{E}-05$ & 23 & 190 & 12 & Regulation of cell migration \\
GO:2000145 & $1.44 \mathrm{E}-06$ & $6.12 \mathrm{E}-05$ & 23 & 195 & 12 & Regulation of cell motility \\
GO:0048870 & $9.75 \mathrm{E}-10$ & $1.81 \mathrm{E}-07$ & 38 & 330 & 12 & Cell motility \\
GO:0042060 & $1.04 \mathrm{E}-10$ & $3.09 \mathrm{E}-08$ & 50 & 485 & 10 & Wound healing \\
\hline
\end{tabular}

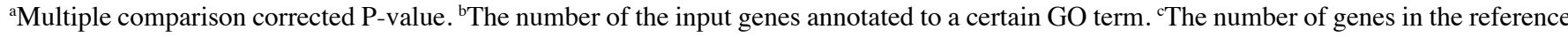
set annotated to a certain GO term.
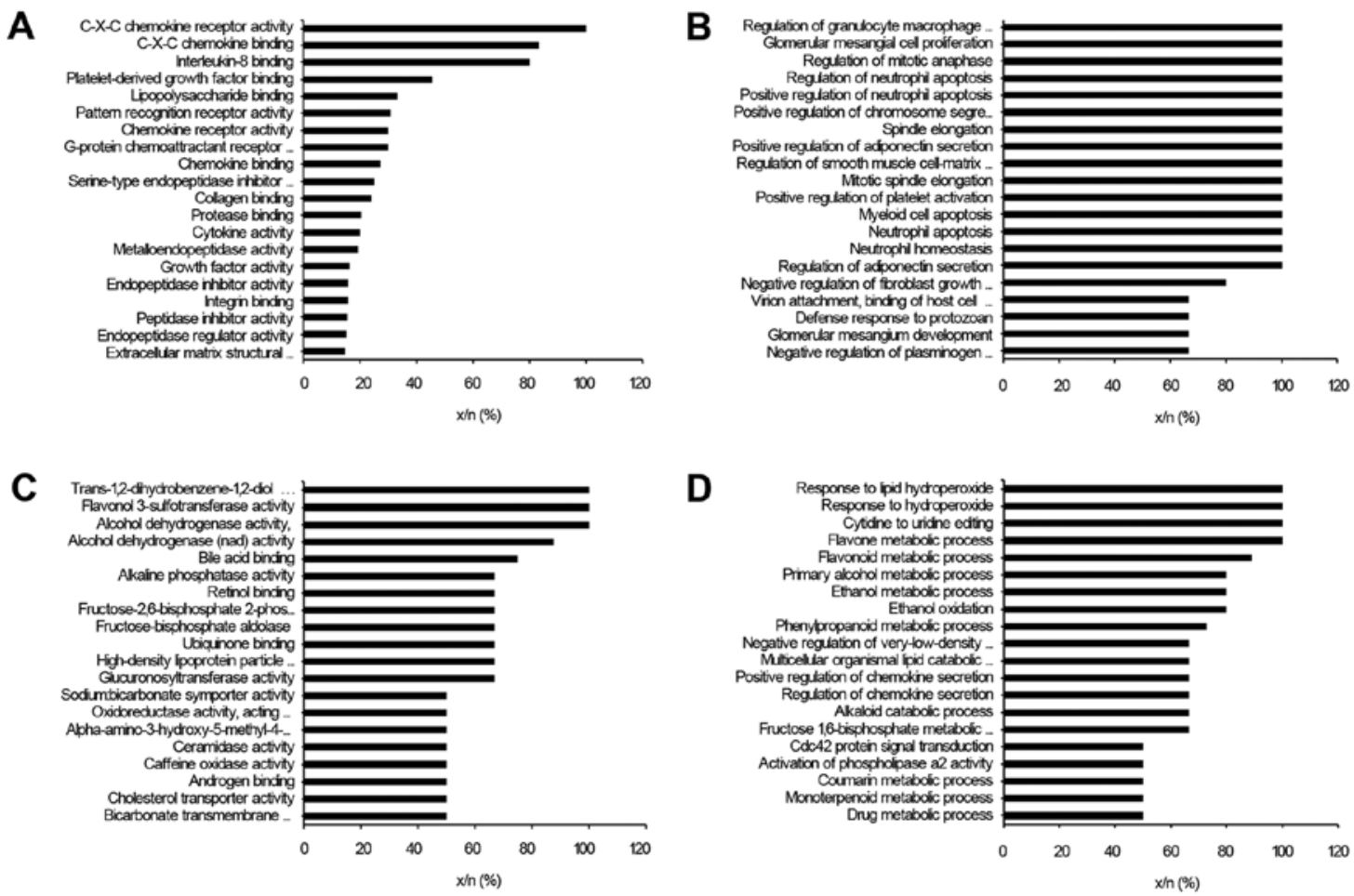

Figure 1. The GO analysis by BiNGO. (A) The upregulated molecular functions in EGC tissues. (B) The upregulated biological process in EGC tissues. (C) The downregulated molecular functions in EGC tissues. (D) The downregulated biological process in EGC tissues. The Information is presented as a percentage of $\mathrm{x} / \mathrm{n}$ ( $x$, the number of genes in a cluster annotated for a certain GO term; $n$, the number of genes in the reference set annotated for a certain GO term).

and E-MTAB-62, into standard scores (z-scores), and then performed hierarchical clustering for the 6 cancers.

\section{Results}

Genome-wide expression analysis. We selected differentially expressed genes (i.e., up- and downregulated genes) from the 27 pairs of EGC tissue and their adjacent normal tissue. The P-value cut-off of 0.05 in t-tests, and the fold-change cut-off of
1.5 or 1/1.5 for up- and downregulated genes, respectively, was used for selection. We identified 556 upregulated genes and 417 downregulated genes. The differentially expressed genes were then fed into function, pathway and cross-experiment analyses to acquire a deeper understanding of the molecular basis of EGC.

Functional enrichment analysis. The BiNGO plug-in on the Cytoscape platform (http://www.cytoscape.org/) was used to explore the molecular function and biological processes in GO. 
Table IV. Pathway enrichment analysis for up- and downregulated genes in gastric cancer tissues.

\begin{tabular}{|c|c|c|c|}
\hline Input genes & Pathways & Count $^{\mathrm{a}}$ & P-value \\
\hline \multirow{23}{*}{$\begin{array}{l}\text { Upregulated } \\
\text { pathways }\end{array}$} & hsa04060, cytokine-cytokine receptor interaction & 38 & 3.61.E-11 \\
\hline & hsa04512, ECM-receptor interaction & 17 & 3.16.E-07 \\
\hline & hsa04110, cell cycle & 19 & 4.21.E-06 \\
\hline & hsa04640, hematopoietic cell lineage & 13 & 2.40.E-04 \\
\hline & hsa04620, Toll-like receptor signaling pathway & 14 & 3.02.E-04 \\
\hline & hsa04062, chemokine signaling pathway & 20 & 3.18.E-04 \\
\hline & hsa04610, complement and coagulation cascades & 11 & 5.85.E-04 \\
\hline & hsa04510, focal adhesion & 19 & 2.00.E-03 \\
\hline & hsa04115, p53 signaling pathway & 10 & 2.11.E-03 \\
\hline & hsa04514, cell adhesion molecules (CAMs) & 14 & 3.72.E-03 \\
\hline & hsa05222, small cell lung cancer & 10 & 8.79.E-03 \\
\hline & hsa04670, leukocyte transendothelial migration & 12 & 1.11.E-02 \\
\hline & hsa05020, prion diseases & 6 & 1.51.E-02 \\
\hline & hsa04621, NOD-like receptor signaling pathway & 8 & 1.53.E-02 \\
\hline & hsa05200, pathways in cancer & 23 & 2.09.E-02 \\
\hline & hsa05332, graft vs. host disease & 6 & 2.34.E-02 \\
\hline & hsa05322, systemic lupus erythematosus & 10 & 2.40.E-02 \\
\hline & hsa05219, bladder cancer & 6 & 3.13.E-02 \\
\hline & hsa04114, oocyte meiosis & 10 & 4.32.E-02 \\
\hline & hsa04650, natural killer cell mediated cytotoxicity & 11 & 5.53.E-02 \\
\hline & hsa04142, lysosome & 10 & 5.97.E-02 \\
\hline & hsa04630, Jak-STAT signaling pathway & 12 & 6.43.E-02 \\
\hline & hsa04914, progesterone-mediated oocyte maturation & 8 & 7.17.E-02 \\
\hline \multirow{22}{*}{$\begin{array}{l}\text { Downregulated } \\
\text { pathways }\end{array}$} & hsa00980, metabolism of xenobiotics by cytochrome P450 & 22 & 3.33.E-18 \\
\hline & hsa00982, drug metabolism & 22 & 7.29.E-18 \\
\hline & hsa00830, retinol metabolism & 19 & 2.76.E-15 \\
\hline & hsa00140, steroid hormone biosynthesis & 13 & 3.63.E-09 \\
\hline & hsa00500, starch and sucrose metabolism & 11 & 2.00.E-07 \\
\hline & hsa00040, pentose and glucuronate interconversions & 8 & 3.57.E-07 \\
\hline & hsa00590, arachidonic acid metabolism & 12 & 3.95.E-07 \\
\hline & hsa00983, drug metabolism & 10 & 2.69.E-06 \\
\hline & hsa00053, ascorbate and aldarate metabolism & 7 & 5.02.E-06 \\
\hline & hsa00591, linoleic acid metabolism & 8 & 1.04.E-05 \\
\hline & hsa00860, porphyrin and chlorophyll metabolism & 8 & 3.32.E-05 \\
\hline & hsa00010, glycolysis/gluconeogenesis & 10 & 4.64.E-05 \\
\hline & hsa00150, androgen and estrogen metabolism & 8 & 7.27.E-05 \\
\hline & hsa03320, PPAR signaling pathway & 7 & 1.41.E-02 \\
\hline & hsa00051, fructose and mannose metabolism & 5 & 1.59.E-02 \\
\hline & hsa00330, arginine and proline metabolism & 6 & 1.78.E-02 \\
\hline & hsa00071, fatty acid metabolism & 5 & 2.74.E-02 \\
\hline & hsa00030, pentose phosphate pathway & 4 & 3.42.E-02 \\
\hline & hsa00350, tyrosine metabolism & 5 & 3.72.E-02 \\
\hline & hsa00920, sulfur metabolism & 3 & 4.53.E-02 \\
\hline & hsa00340, histidine metabolism & 4 & 5.00.E-02 \\
\hline & hsa00480, glutathione metabolism & 5 & 5.54.E-02 \\
\hline
\end{tabular}

${ }^{a}$ Count represents the number of input genes assigned to the KEGG pathway.

The functions of the upregulated genes of EGC tissues were significantly associated with $\mathrm{C}-\mathrm{X}-\mathrm{C}$ and other chemokine-related signaling, interleukin-8 binding, growth factor binding, collagen binding, and the extracellular matrix (ECM) (Fig. 1A). Moreover, the biological processes involved in the upregulated genes in the EGC tissues were strongly related to 
cell proliferation, mitosis, apoptosis and cell-matrix adhesion (Fig. 1B). Additionally, wound healing terms, cell migration terms and cell motility terms were also listed in the upregulated genes with statistical significance (Table III). Since cell migration, cell motility and wound healing are typically observed in late-stage, metastatic cancer, this may indicate that EGC tissues could possess intrinsic aggressiveness, despite their early detection. Conversely, the downregulated genes were strongly linked to oxidoreductase activity (e.g., oxidoreductase activity acting on the $\mathrm{CH}$ or $\mathrm{CH} 2$ groups, quinones) in $\mathrm{GO}$ molecular function (Fig. 1C). Furthermore, the downregulated genes were enriched in various terms related to metabolic processes (e.g., flavone and flavonoid metabolic pathways) in GO biological processes (Fig. 1D). The GO terms of the downregulated genes clearly indicate dysregulation of metabolism in EGC, which is one of the emerging cancer hallmarks (14).

Pathway enrichment analysis. The DAVID tool (http://david. abcc.ncifcrf.gov/) was used to inspect the KEGG biological pathways associated with the differently expressed genes in EGC.

The upregulated genes in EGC tissues were intrinsically associated with cytokine-cytokine receptor interactions, ECM-receptor interactions, the cell cycle, hematopoietic cell lineage and Toll-like receptor signaling pathways (Table IV). In addition, focal adhesion and cell adhesion molecule pathways were highlighted. Thus, similar to the functional enrichment analysis, upregulated pathways in these tumor tissues suggest a strong potential for cell motility and metastasis, despite early detection. In contrast, the downregulated genes in the EGC tissues were strongly associated with xenobiotics-, drug-, retinol-, starch- and sucrose-related metabolism, steroid hormone biosynthesis, as well as pentose and glucuronate interconversion pathways (Table IV).

The expression of MMPs in EGC tissue. Our functional and pathway analyses demonstrated that the significantly upregulated genes in EGC tissues are associated with cell migration and metastasis, events typical of late-stage cancer. To verify our findings, we further analyzed the expression pattern of matrix metalloproteinases (MMPs), which are well known cell migration-related genes. MMPs are also known to play critical roles in the regulation of cell invasion by ECM proteolysis, as well as by processing cytokine precursors in the chemokine network.

We analyzed the expression pattern of 7 MMPs (MMP1, $-3,-7,-9,-10,-12$ and -13) within the upregulated gene data in EGC tissues. As expected, and consistent with the microarray data where MMPs were upregulated 1.56- to 8.68-fold (Fig. 2A), RT-PCR indicated that MMP mRNA expression was highly upregulated in the patients' EGC tissues (Fig. 2B).

Cross-experimental analysis. In order to investigate similar molecular signatures between EGC and other cancer types, we compared our data of differentially expressed genes with a public gene expression signature warehouse, L2L. This revealed that the upregulated genes in EGC most significantly correlated with the gene expression signature of ER $\alpha$-negative breast cancer (Table V). As summarized in Table V, the upregulated genes in EGC were also similar to the gene expression
A

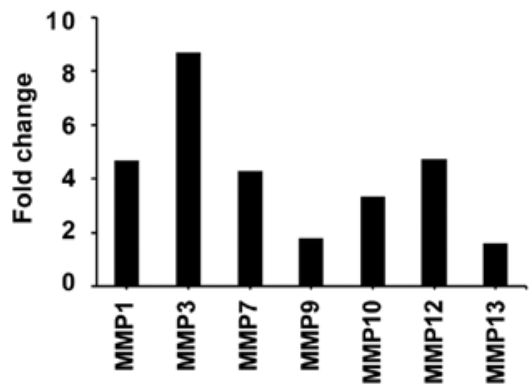

B

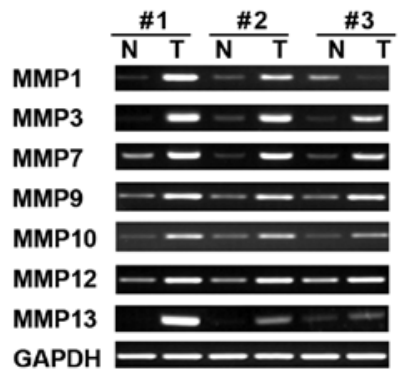

Figure 2. The expression of MMPs in EGC tissues. (A) Expression levels of MMPs genes in microarray data. The vertical axis represents fold-change of the cancer tissues over normal tissues. (B) mRNA expression of MMPs using RT-PCR. Three pairs of non-cancerous and tumor tissues from the microarray analysis were used. (N, adjacent non-cancerous gastric tissue; T, EGC tissue).

signature related to an undifferentiated cancer status (cancer_ undifferentiated_meta_up: 69 genes commonly upregulated in undifferentiated cancer relative to well-differentiated cancer, from a meta-analysis of the OncoMine gene expression database), stemness (stemcell_embryonic_up: enriched in mouse embryonic stem cells, compared to differentiated brain and bone marrow cells) and survival (dox_resist_gastric_up: upregulated in gastric cancer cell lines resistant to doxorubicin, compared to parent chemosensitive lines). Together, the EGC tissues reflect various facets of cancer-related phenotypes, viz., strong survival, stem-like and morphology.

The same L2L analysis was applied to the downregulated genes in EGC (Table V). Interestingly, epigenetic-related cancer gene expression signature terms (5azac_hepg2_up and 5azac-tsa_hepg2_up in Table V) were highly ranked. This suggests that global alterations in DNA methylation and histone modification occur in EGCs, as it does in other cancers.

Hierarchical clustering of the EGC tissues and other cancers. To validate the result of the $\mathrm{L} 2 \mathrm{~L}$ analysis showing a relationship between EGC and ER $\alpha$-negative breast cancer, we performed a hierarchical clustering analysis. The expression datasets of the differently expressed genes in EGC (556 upregulated gene symbols and 417 downregulated gene symbols), ER $\alpha$-negative breast cancer and 4 additional cancers (small cell lung cancer, LNCaP prostate cancer cell lines, bladder cancer and Ewing sarcoma) were used in an unsupervised hierarchical clustering analysis. As in the L2L analysis, the results indicated that EGC correlated most closely with the ER $\alpha$-negative cancer than with the other 4 cancers (Fig. 3).

When we inspected the expression levels (z-scores) of the 7 MMP genes (Fig. 4), the results indicated that the ER $\alpha$-negative 


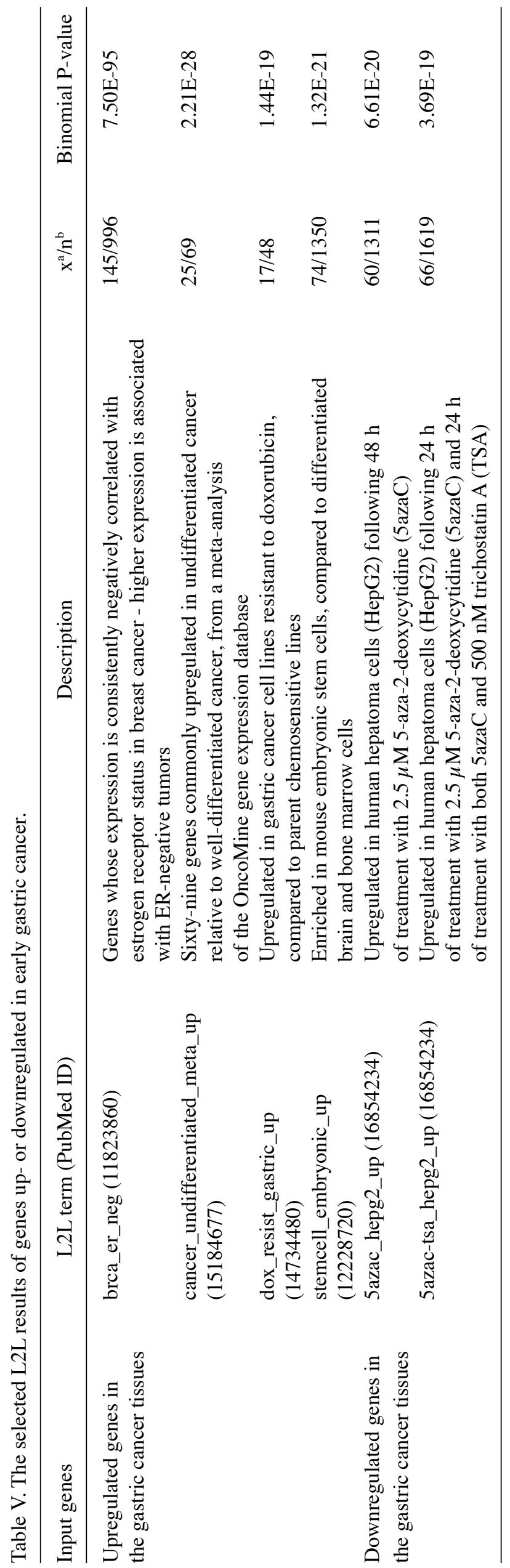

Table VI. Comparisons with other GC sources in terms of upregulated EGC-related genes.

\begin{tabular}{llccc}
\hline Refs. & \multicolumn{1}{c}{$\begin{array}{c}\text { EGC } \\
\text { classification }\end{array}$} & $\mathrm{n}^{\mathrm{a}}$ & $\mathrm{x}^{\mathrm{b}}$ & $\begin{array}{c}\text { Significance } \\
(\mathrm{P}-\text {-value })^{\mathrm{c}}\end{array}$ \\
\hline$(2)$ & \multicolumn{1}{c}{-} & 488 & 83 & $<2.2 \mathrm{E}-16$ \\
$(7)$ & $\begin{array}{l}\text { Well-differentiated (WD) } \\
\text { and moderately }\end{array}$ & 170 & 62 & $<2.2 \mathrm{E}-16$ \\
& $\begin{array}{l}\text { differentiated (MD) } \\
\text { (8) }\end{array}$ & & & \\
& $\begin{array}{l}\text { AJCC staging I and II } \\
\text { (TNM staging) }\end{array}$ & 118 & 15 & $1.601 \mathrm{E}-06$ \\
& & &
\end{tabular}

${ }^{a}$ The number of upregulated genes in the cancer according to the references. ${ }^{b}$ The number of common upregulated genes (intersection)

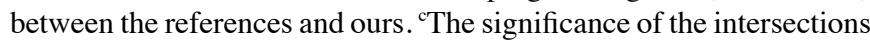
between our EGC upregulated genes and the studies were calculated. Fisher's exact test, based on the randomization model, was used to obtain the P-values of the intersections from a hyper-geometric distribution. The smaller the P-value, the more significant the agreement between the previous study and our EGC study. The total number of gene symbols used in the Fisher's exact test is 19,211 (HUGO Gene Nomenclature Committee).

cancer, above all other observed cancers, showed the most similar expression patterns for the 7 MMPs. Overall, the hierarchical clustering was consistent with the cross-experimental analysis and strongly supported the molecular similarity between EGC and ER $\alpha$-negative breast cancer in terms of carcinogenesis.

\section{Discussion}

We analyzed the microarray data generated from pairs of tumor tissue and their adjacent non-cancerous tissue, obtained from 27 EGC patients. The gene expression data were subjected to functional and pathway analyses, as well as gene expression signature comparison (cross-experiment analysis). This led to 2 novel findings: i) the functional and pathway analyses suggested that metastasis-related biological processes may already be highly expressed even in the early stage of gastric cancer, and ii) the gene expression pattern of EGC is closely aligned to that of ER $\alpha$-negative breast cancer.

We also compared the differentially expressed genes in our EGC tissues with other 3 previously published gene expression studies $(2,7,8)$. We found that the upregulated genes in our study significantly overlapped with the upregulated genes in the EGC groups of the 3 earlier studies $(2,7,8)$, under a randomization model (Table VI). Recently, Vecchi et al suggested a carcinogenesis model (2) in which the transition from normal mucosa to EGC is accompanied by cell cycle upregulation; our pathway analysis results (hsa04110, cell cycle in Table IV) is consistent with this model. Interestingly, AGC functions (cell migrationand ECM-related functions), suggested by the Vecchi model were also revealed in our EGC data, again indicating that EGC actually harbors gene expression events that are usually observed in the later stages of cancer, such as AGC.

Based on our functional and pathway analyses, the upregulated genes in the EGC tissues were highly enriched for 


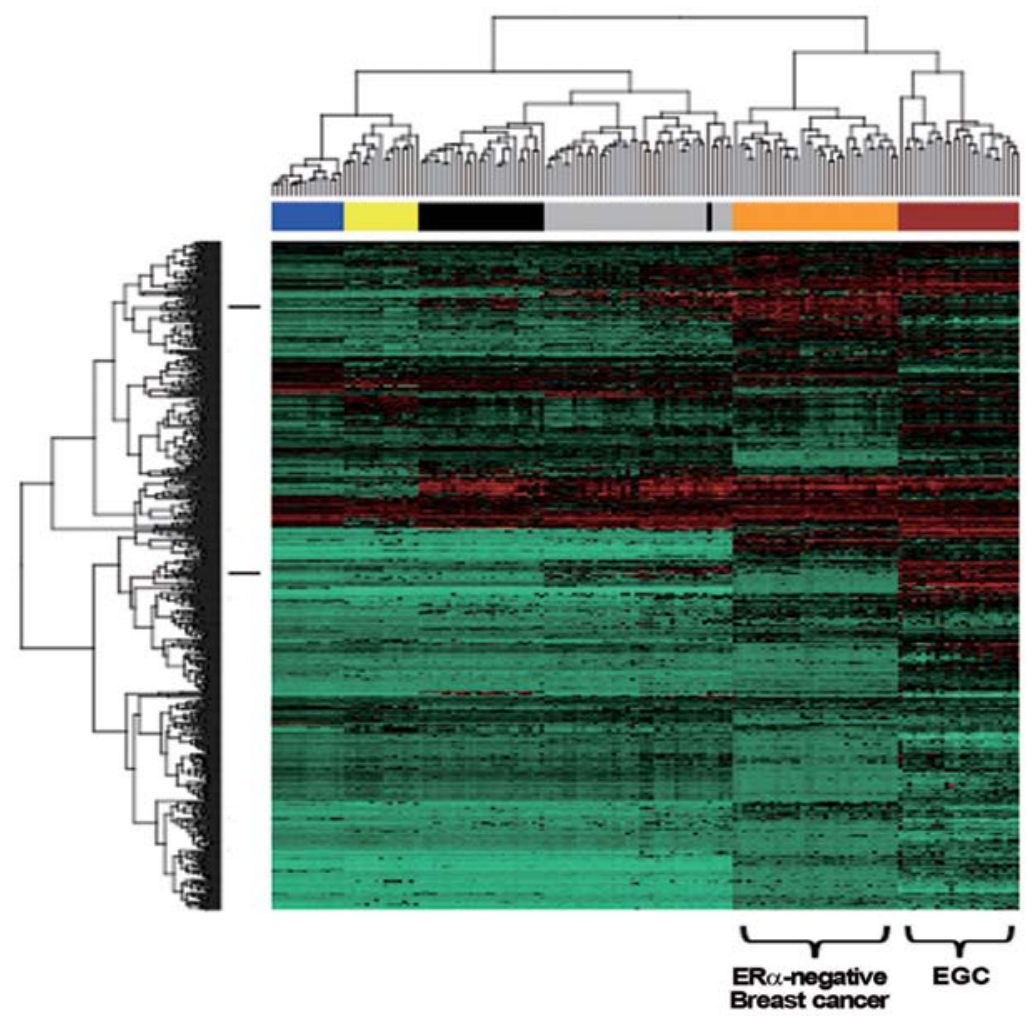

Figure 3. Hierarchical clustering. Genes up- or downregulated in EGC as well as 5 independent cancer types were used in the hierarchical clustering analysis. Each cancer type is presented with the following column side-bars: EGC (brown), ER $\alpha$-negative breast cancers (orange), bladder cancer (grey), Ewing sarcoma (black), small cell lung cancers (yellow) and LNCaP prostate cancer cell lines (blue). Seven MMP genes are presented with row side-bars.

A

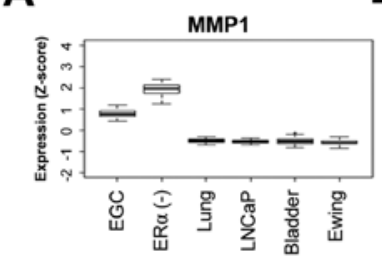

D

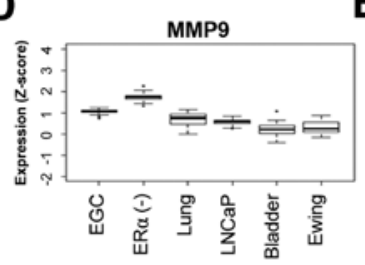

G

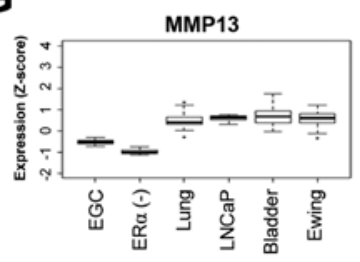

B

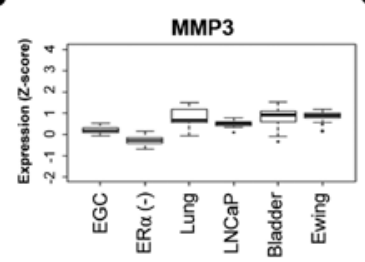

E

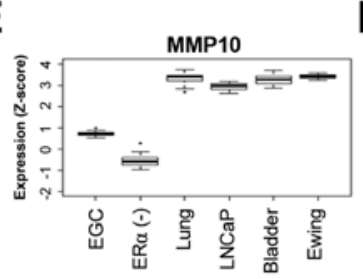

C

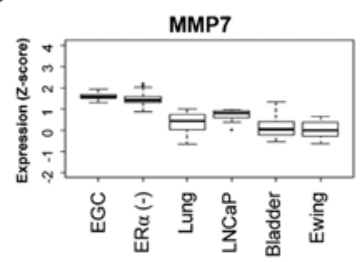

$\mathbf{F}$

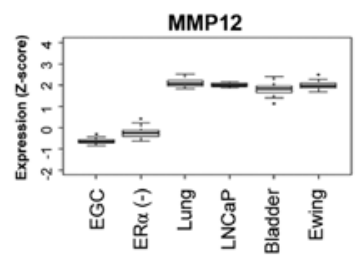

Figure 4. The expressions (Z-scores) of 7 MMP genes. Z-score zero corresponds to the mean of all expressions in each cancer type. (EGC, the EGC tissues; ERo $(-), E R \alpha$-negative breast cancer; Lung, lung small cell cancer; LNCaP, LNCaP prostate cancer cell lines; Bladder, bladder cancer; and Ewing, Ewing sarcoma).

genes involved in cell proliferation, chemokine/growth factor signaling and cell migration. The computational implication is, in fact, closely related to MMP activity, as MMP substrates include growth factor/chemokine precursors and E-cadherin
$(15,16)$. We validated the upregulation of the 7 MMPs in the EGC tissues by RT-PCR. This result suggests that the activation of multiple MMPs may be involved in the early stage of cancer. The suggestion is noteworthy, when considering that 
A

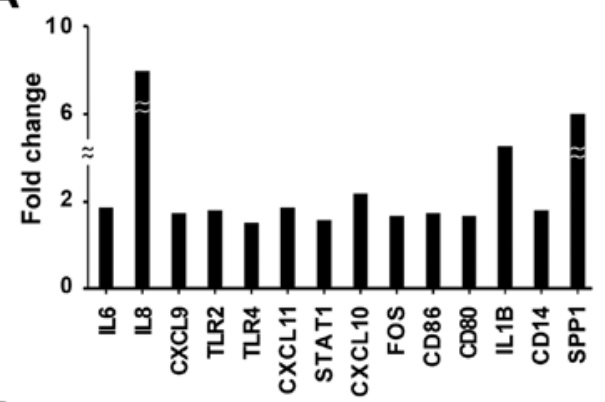

B

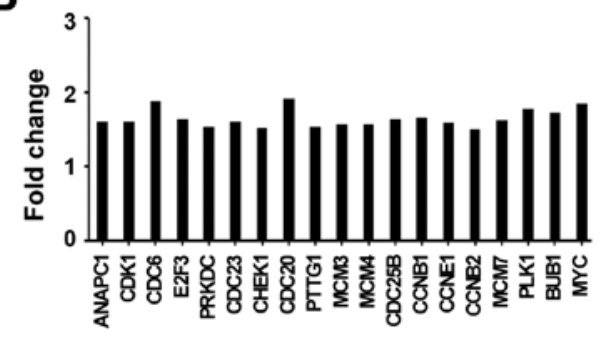

Figure 5. The upregulated genes belonging to the KEGG TLR signaling pathway (A) and cell cycle pathway (B) in our EGC tissues.

the roles of multiple MMPs were mainly reported in latestage gastric cancer $(2,17)$. It is also interesting to note that 6 (MMP1, -3, -7, -10, -12 and -13) of the 7 MMPs are clustered at $11 \mathrm{q} 22$, implying that epigenetic events could be involved in the upregulation of the clustered MMPs (18).

Additionally, we found that the gene expression pattern in EGC tissues resembles the pattern of the ER $\alpha$-negative breast cancer transcriptome. Since ER $\alpha$-negative breast cancer clusters with EGC (Fig. 3), the similarity suggests that these two cancers may share common molecular features. Recent breast cancer studies $(19,20)$ reported that high expression of cyclooygenase-2 (Cox-2), encoded by PTGS2, is associated with poor survival in ER $\alpha$-negative breast cancer patients, when compared to ER $\alpha$-positive breast cancers. Interestingly, Cox-2 is highly involved in the inflammation-associated carcinogenesis of the gastrointestinal tract. In particular, H. pylori-infected gastric epithelial cells can experience malignant transformation via Toll-like receptor (TLR) signaling that induces Cox-2, followed by activation of cell proliferation (21). In fact, our pathway analysis in EGC showed upregulation of the KEGG TLR signaling pathway and cell cycle pathway (Table IV, Fig. 5). Our EGC also showed a markedly increased expression of PTGS2 (5.74-fold-change). Thus, the similarity between EGC and ER $\alpha$-negative breast cancer may come from identical subsets of immune response-related signaling between the microenvironments of the tumors.

In conclusion, we have analyzed the differentially expressed genes in EGC patients using an integrative systematic approach. We found that genes highly expressed in EGC are involved in cell migration- and metastasis-related functions typically observed in late-stage cancer. Also, EGC may be intrinsically similar to ER $\alpha$-negative breast cancer, by sharing immunerelated signaling events, which is further dissected in both cancer types. The functional roles of the downregulated genes in EGC carcinogenesis remain to be elucidated in future.

\section{Acknowledgements}

This study was funded by Intramural Research Grants of the National Cancer Center (NCC 1210460-1).

\section{References}

1. Parkin DM, Bray F, Ferlay $\mathbf{J}$ and Pisani P: Global cancer statistics, 2002. CA Cancer J Clin 55: 74-108, 2005.

2. Vecchi M, Nuciforo P, Romagnoli S, et al: Gene expression analysis of early and advanced gastric cancers. Oncogene 26: 4284-4294, 2007.

3. Crew KD and Neugut AI: Epidemiology of gastric cancer. World J Gastroenterol 12: 354-362, 2006.

4. Nam SY, Choi IJ, Park KW, et al: Effect of repeated endoscopic screening on the incidence and treatment of gastric cancer in health screenees. Eur J Gastroenterol Hepatol 21: 855-860, 2009.

5. Takeno A, Takemasa I, Doki Y, et al: Integrative approach for differentially overexpressed genes in gastric cancer by combining large-scale gene expression profiling and network analysis. $\mathrm{Br} \mathrm{J}$ Cancer 99: 1307-1315, 2008.

6. Yamada Y, Arao T, Gotoda T, et al: Identification of prognostic biomarkers in gastric cancer using endoscopic biopsy samples. Cancer Sci 99: 2193-2199, 2008.

7. Cui J, Li F, Wang G, Fang X, Puett JD and Xu Y: Gene-expression signatures can distinguish gastric cancer grades and stages. PLoS One 6: e17819, 2011.

8. Economescu MC, Necula LG, Dragu D, et al: Identification of potential biomarkers for early and advanced gastric adenocarcinoma detection. Hepatogastroenterology 57: 1453-1464, 2010.

9. Maere S, Heymans K and Kuiper M: BiNGO: a cytoscape plugin to assess overrepresentation of gene ontology categories in biological networks. Bioinformatics 21: 3448-3449, 2005.

10. Kanehisa M, Goto S, Furumichi M, Tanabe M and Hirakawa M: KEGG for representation and analysis of molecular networks involving diseases and drugs. Nucleic Acids Res 38: D355-D360, 2010.

11. Newman JC and Weiner AM: L2L: a simple tool for discovering the hidden significance in microarray expression data. Genome Biol 6: R81, 2005.

12. Enerly E, Steinfeld I, Kleivi K, et al: miRNA-mRNA integrated analysis reveals roles for miRNAs in primary breast tumors. PLoS One 6: e16915, 2011.

13. Lukk M, Kapushesky M, Nikkila J, et al: A global map of human gene expression. Nat Biotechnol 28: 322-324, 2010.

14. Hanahan D and Weinberg RA: Hallmarks of cancer: the next generation. Cell 144: 646-674, 2011.

15. Roy R, Yang J and Moses MA: Matrix metalloproteinases as novel biomarkers and potential therapeutic targets in human cancer. J Clin Oncol 27: 5287-5297, 2009.

16. Foley J, Nickerson NK, Nam S, et al: EGFR signaling in breast cancer: bad to the bone. Semin Cell Dev Biol 21: 951-960, 2010.

17. Murray GI, Duncan ME, Arbuckle E, Melvin WT and Fothergill JE: Matrix metalloproteinases and their inhibitors in gastric cancer. Gut 43: 791-797, 1998.

18. Clark IM, Swingler TE, Sampieri CL and Edwards DR: The regulation of matrix metalloproteinases and their inhibitors. Int J Biochem Cell Biol 40: 1362-1378, 2008.

19. Glynn SA, Prueitt RL, Ridnour LA, et al: COX-2 activation is associated with Akt phosphorylation and poor survival in ER-negative, HER2-positive breast cancer. BMC Cancer 10: 626, 2010.

20. Witton CJ,Hawe SJ, Cooke TG and Bartlett JM: Cyclooxygenase 2 (COX2) expression is associated with poor outcome in ER-negative, but not ER-positive, breast cancer. Histopathology 45: 47-54, 2004.

21. Fukata M and Abreu MT: Role of Toll-like receptors in gastrointestinal malignancies. Oncogene 27: 234-243, 2008. 